Technological University Dublin

2004-05-10

\title{
Investigation of the Diffusion Processes in Self-processing Acrylamide-based Photopolymer System
}

\author{
Izabela Naydenova \\ Technological University Dublin, izabela.naydenova@tudublin.ie \\ Raghavendra Jallapuram \\ Technological University of Dublin \\ Robert Howard \\ Technological University of Dublin
}

See next page for additional authors

Follow this and additional works at: https://arrow.tudublin.ie/cieoart

Part of the Condensed Matter Physics Commons, and the Optics Commons

\section{Recommended Citation}

Naydenova, I. et al. (2004) Investigation of the Diffusion Processes in Self-Processing Acrylamide-Based Photopolymer System. Applied Optics, Vol. 43 no.14, pp.2900-2905. doi:10.1364/A0.43.002900

This Article is brought to you for free and open access by the Centre for Industrial and Engineering Optics at ARROW@TU Dublin. It has been accepted for inclusion in Articles by an authorized administrator of ARROW@TU Dublin. For more information, please contact arrow.admin@tudublin.ie, aisling.coyne@tudublin.ie, gerard.connolly@tudublin.ie.

Funder: Facility for Optical Characterization and Spectroscopy at Technological University of Dublin

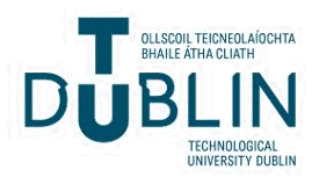




\section{Authors}

Izabela Naydenova, Raghavendra Jallapuram, Robert Howard, Suzanne Martin, and Vincent Toal 


\title{
Investigation of the diffusion processes in self-processing
} acrylamide-based photopolymer system

\author{
Izabela Naydenova ${ }^{1}$, Raghavendra Jallapuram ${ }^{1}$, Robert Howard ${ }^{2}$, \\ Suzanne Martin ${ }^{1}$, Vincent Toal ${ }^{1,2}$ \\ 1) Centre for Industrial and Engineering Optics, Dublin Institute of Technology, Kevin Street, Dublin 8 \\ 2) School of Physics, Dublin Institute of Technology, Kevin Street, Dublin 8, Ireland \\ e-mail: izabela.naydenova@dit.ie \\ OCIS codes: 090.2900; 090.7330; 290.1990
}

\begin{abstract}
Results from the investigation of the diffusion processes in a dry acrylamide-based photopolymer system are presented. The investigation is carried out in the context of experimental work on optimization of the high spatial frequency response of the photopolymer. Tracing the transmission holographic grating dynamics at short times of exposure is utilized to measure diffusion coefficients. The results reveal that two different diffusion processes contribute with opposite sign to the refractive index modulation responsible for the diffraction grating build up. Monomer diffusion from dark to bright fringe areas increases the refractive index modulation. It is characterized with diffusion constant $\mathrm{D}_{0}=1 \cdot 6 \cdot 10^{-7} \mathrm{~cm}^{2} / \mathrm{s}$. A second diffusion process takes place during the recording. It decreases the refractive index modulation and we ascribe it to diffusion of short chain polymer molecules and/or radicals from bright to dark fringe areas. The estimated diffusion coefficient for this process is $\mathrm{D}_{0}=6.35 .10^{-10} \mathrm{~cm}^{2} / \mathrm{s}$. The presence of the second process could be responsible for poor high spatial frequency response of the investigated photopolymer
\end{abstract}


system. Comparison with the diffusion in photopolymer systems known for their good response at high spatial frequencies shows that both investigated diffusion processes measured here occur in a much faster time scale.

\section{Introduction}

Photopolymers represent a class of photosensitive materials attracting scientific and industrial interest because of their high sensitivity, large dynamic range, easy processing and relatively low cost. Development of dry self-processing photopolymer systems makes photopolymers suitable for a large variety of applications - design of optical elements ${ }^{1,2}$, real-time interferometry ${ }^{3,4}$ and write once optical memories ${ }^{5-7}$. The acrylamide - based photopolymer developed in our group ${ }^{2,8}$ has excellent characteristics for transmission holograms recording. In addition to high sensitivity and diffraction efficiency, in normal use it suffers negligible shrinkage during and after the holographic recording in contrast to the data reported for other photopolymer systems ${ }^{9}$. A disadvantage of this photopolymer system in its current formulation is its poor response at high spatial frequency holographic recording. To improve the high special frequency response a thorough understanding of the holographic recording mechanism in this particular system is required. Theoretical models describing holographic grating formation in different photopolymer systems predict that the monomer diffusion rate is a crucial factor for its performance during holographic recording. In the present work we focus our research on investigation of the monomer diffusion process in the acrylamide-based photopolymer system and we compare it with other photopolymers that are known for their good response in reflection mode of recording. To best of our knowledge we present here the first independent measurement of the diffusion constants characterizing the diffusion processes in this dry acrylamide - based photopolymer system. The mechanisms of holographic grating 
formation and poor high spatial frequency response in relation to measured fast diffusion time constants are discussed.

\section{Theory}

Photochemical reactions during the holographic recording in photopolymer system

The basic formulation of the dry photopolymer system contains photoinitiator and monomer that are dispersed in a binder matrix. In the investigated acrylamide-based photopolymer system photoinitiation is a two step process. Upon illumination of the photopolymer with light of appropriate wavelength the sensitizing dye absorbs a photon and reacts with an electron donor to produce free radicals (initiation step). These initiate polymerization where the light was absorbed. Chain propagation or termination follows the initiation. During the propagation reaction free radicals and monomer molecules interact and the polymer chains grow. At the termination step two free radicals interact and a polymer chain stops growing. Currently accepted models describe the recorded pattern formation as a result of changes in the density and the molecular polarizability, which accompany the polymerization. The magnitude of the refractive index change is dependent on the chain length achieved.

\section{Diffusion model for holographic grating formation in photopolymer system}

The diffusion models ${ }^{10-15}$ predict that the key factor that controls dynamics and final properties of the recorded holographic grating (refractive index space profile and modulation) is proportional to the ratio of polymerization rate and monomer diffusion rate. Both parameters are strongly 
dependent on the nature of the photopolymer system and having information about them one can find the optimal conditions for holographic recording. Two different regimes of holographic recording at given spatial frequency can be distinguished with respect to the ratio of the diffusion and polymerization rate. When the polymerization rate is slower than the diffusion rate, the grating profile closely resembles the sinusoidal recording interference pattern and a high saturation value of the refractive index modulation can be achieved. When the monomer diffusion rate is slower than the polymerization rate deviation from the sinusoidal profile of the grating is observed and the diffraction efficiency at saturation is lower. The monomer diffusion rate is characteristic for a given photopolymer system. In a simplified picture when the diffusion dependence on the degree of polymerization is not considered the diffusion time will be constant at given spatial frequency. The polymerization rate, however depends on the recording intensity. By changing the recording intensity one can control the polymerization rate and in such a way to switch between the two regimes - effectively slow diffusion compared to the polymerization rate at high intensity and effectively fast diffusion when the intensity and the polymerization rate are low. There is little data available for diffusion constants in photopolymer systems. In most of it the monomer diffusion rate is relatively slow $\left(6.51 .10^{-11} \mathrm{~cm}^{2} / \mathrm{s}\right.$ for Omnidex DuPont photopolymers ${ }^{11}$ and $3.57 .10^{-14} \mathrm{~cm}^{2} / \mathrm{s}$ for the system investigated by Colvin et $\mathrm{al}^{12}$ ) and increasing the recording intensity is not favorable for high holographic performance of such materials.

Modeling the diffraction grating kinetics in continuous mode ${ }^{13-15}$ can only supply information about the ratio between both rates and in most of the cases the diffusion rates reported are based on assumed polymerization rates. In order to get the information required to optimize our photopolymer system's response during the holographic recording, a separate determination of the diffusion and polymerization rate is necessary. The characterization of a given photopolymer 
system is complicated by the fact that both the diffusion and the polymerization rate depend on the monomer concentration. This leads to a time dependence of both rates during the holographic recording. In the present investigation we have focused our attention on the estimation of the initial diffusion constant $\mathrm{D}_{0}$ using short exposure time for recording holographic gratings with low diffraction efficiency (in order of 1\%).

\section{Experimental}

\section{Materials}

The photosensitive layer was prepared as previously described ${ }^{3}$. Briefly, $2 \mathrm{ml}$ of triethanolamine was added to $17.5 \mathrm{ml}$ stock solution of polyvinyl alcohol (PVA) $(10 \% \mathrm{w} / \mathrm{w})$. Then the monomer, $1 \mathrm{~g}$ acrylamide, was added. In some of the samples a second monomer was used as a crosslinking agent. In these samples $0.32 \mathrm{~g}-\mathrm{N}, \mathrm{N}$ Methylene bisacrylamide and $0.48 \mathrm{~g}$ acrylamide were used. Finally, 4ml of Erythrosin B dye was added (stock solution concentration - 1.1mM). The solution was made up to $25 \mathrm{ml}$ by adding distilled water. $2 \mathrm{ml}$ of this solution were spread on $50 \times 50 \mathrm{~mm}$ plate. The samples were dried for 36 - 48 hours. Sample thickness after drying was $150 \mu \mathrm{m}$.

\section{Measurement of monomer diffusion coefficient}

A method described by Colvin et $\mathrm{al}^{12}$ was utilized to measure the monomer diffusion coefficient. We recorded a transmission grating build-up during and after a short exposure (the recording time must be faster than the expected monomer diffusion time). We ensure that the exposure is 
small enough so that we can assume the diffusion coefficient is not spatially modulated. Transmission gratings with diffraction efficiency of the order of few percent were recorded using $\mathrm{NdYVO}_{4}$ laser (Verdi 05) $\lambda=532 \mathrm{~nm}$. Recording times were in $0.1-2 \mathrm{~s}$ range and the total recording intensity was varied between 3.5 and $70 \mathrm{~mW} / \mathrm{cm}^{2}$. A He $-\mathrm{Ne}$ laser, $\lambda=633 \mathrm{~nm}$, was used for non-destructive real - time observation of grating formation. The photopolymer absorption is negligible at $633 \mathrm{~nm}$ and the investigated gratings are considered as phase gratings, i.e. due to photoinduced change in refractive index. Transmission gratings at different spatial frequencies in the range $200-20001 / \mathrm{mm}$ were investigated. Diffracted signals in the +1 order were recorded using a Newport Model 840 optical power meter. A Laplace Instruments data acquisition system was used to transfer the signal from the photodetector to a computer. Time resolution of the data acquisition system was $5 \mathrm{~ms}$.

The refractive index modulation $\Delta n$ was calculated using the measured diffraction efficiency $\eta$ according to Kogelnik's theory ${ }^{16}$ in the following way:

$$
\Delta n=\frac{\lambda \cos \theta \cdot A r c \sin \sqrt{\eta}}{\pi \cdot d .},
$$

where $d$ is the sample thickness, $\lambda$ - reconstructing beam wavelength and $\theta$ - reading beam incidence angle. After taking into account the level of refractive index modulation ${ }^{20,21}$ we have estimated that even at the lowest reported spatial frequency $(100 \mathrm{l} / \mathrm{mm})$ equation (1) is applicable with a good approximation. The curves representing the time dependence of the refractive index change were fitted with Microcal Origin software applying the Levenberg-Marquardt method for minimizing the chi-square value. The diffusion time $\tau_{\mathrm{d}}$ at given spatial frequency was extracted from the fitting results. Using the relation ${ }^{11,14}$ 


$$
\tau_{d}=\frac{1}{D_{0} K^{2}}
$$

where $\mathrm{K}=2 \pi / \Lambda, \Lambda$ - fringe spacing, after linear fit of the diffusion time dependence on $1 / \mathrm{K}^{2}$, the diffusion constant $\mathrm{D}_{0}$ was calculated.

\section{Results and discussion}

Preliminary investigation ${ }^{8}$ of the presented photopolymer system suggests that the diffusion processes must happen in much faster time scale than in other dry photopolymer systems. In order to better resolve the refractive index changes due to diffusion our investigation began at low spatial frequency of recording. The gratings were recorded for $0.1 \mathrm{~s}$ with recording exposure $40 \mathrm{~mW} / \mathrm{cm}^{2}$. A characteristic curve for the refractive index modulation at a spatial frequency of $200 \mathrm{l} / \mathrm{mm}$ is presented in Figure 1. The maximum diffraction efficiency of the recorded grating was $<1 \%$. Fresnel reflection losses were taken into account by multiplication of the diffraction efficiency expression by an appropriate factor. After the recording beams had been switched off (the moment is shown with an arrow in the figure) an initial increase (labeled as postprocess 1) followed by a decrease (postprocess 2) in the refractive index modulation were observed. Both processes can be characterized by single time constants after fitting the recorded kinetics with a two-exponential function. Data fit using three or four exponential functions was also carried out but the fit results in these cases were not characterised by a better chi-square value. As has been observed in other photopolymer systems ${ }^{17,} 18$ illumination with homogeneous light (postprocess 3 in Fig. 1) increased the diffraction efficiency. For postexposure a third beam, 
different than the recording beams, was used so the effects related to illumination under Bragg angle, generation of a diffracted wave and existence of an interference pattern due to superposition of these two waves, were avoided. An increase in the diffraction efficiency after illumination with a single beam was observed for the gratings with very low diffraction efficiencies (a few percent) and also for those with more than $30 \%$ diffraction efficiency. The illumination with homogeneous light has a similar effect at low $(100 \mathrm{l} / \mathrm{mm})$ and at high $(20001 / \mathrm{mm})$ spatial frequency of recording. This effect confirms that the recorded grating is not a result of polymerization only, otherwise homogeneous polymerization would erase the grating. Some additional process, accompanying the polymerization must take place during the recording in the photopolymer and must have a positive contribution to the final refractive index modulation. We ascribe this process to mass transport of monomer from dark to bright fringe areas due to a concentration gradient created during illumination with spatially modulated field. The increase in the refractive index modulation during postprocess 3 can be explained by the existence of a second spatial modulation of the refractive index that creates a phase grating shifted by $\pi$ with respect to the main grating. The origin is the conversion of the acrylamide double to single bond during the polymerization. The lower polarizability of the single bonds would, if this effect were considered, reduce the refraction index in exposed area ${ }^{19}$. Without post exposure, the grating due to the mass transport (higher refractive index in the bright fringe areas) and the grating due to bond conversion (lower refractive index in bright fringe areas) are superimposed and the final grating will have diffraction efficiency corresponding to the difference between the two gratings. When the sample is illuminated with homogeneous light the grating due to the bond conversions is erased and the effective diffraction efficiency observed increases (Fig.1 postprocess 3). 
In order to check whether the initial positive contribution to the refractive index modulation (postprocess 1) is related to monomer diffusion, gratings at different spatial frequencies were recorded (100 to $2000 \mathrm{l} / \mathrm{mm}$ ). The dynamics of the gratings recorded at 200, 500 , and $1000 \mathrm{l} / \mathrm{mm}$ after illumination for $0.5 \mathrm{~s}$ with intensity $10 \mathrm{~mW} / \mathrm{cm}^{2}$ show that in this range there is no dependence of the rise time on the spatial frequency. Only at very large fringe spacing (> $5 \mu \mathrm{m}$ ) was the dependence of the dynamics of the initial process on the spatial frequency observed. Data for 100 and 200 1/mm are shown in Fig.2. It is seen from the figure that the initial rate of increase in the refractive index modulation is lower at $100 \mathrm{l} / \mathrm{mm}$. The dependence of the initial rate of increase on the spatial frequency was better observed in the samples containing $\mathrm{N}, \mathrm{N}$ Methylene-bisacrylamide as all postprocesses were much slower in this sample (Fig.3). Here the rate of increase clearly slowed as the spatial frequencies decreased. As the polymerization rate itself should not depend on the spatial frequency (at such low \% conversions) monomer diffusion from dark to bright fringe areas must be the main contributor to this increase of the diffraction efficiency. At higher spatial frequencies the diffusion distance is shorter, so any diffusion related process would be expected to be faster. Similar increase in the diffraction efficiency of the recorded gratings at short exposure times has been observed in other photopolymer systems ${ }^{11,12}$.

Analyzing the spatial frequency dependence of the dynamics of postprocess 2 in the same recorded gratings (Fig. 3) we observed that increasing the spatial frequency speeds up the decay in refractive index modulation. The same dependence on the spatial frequency was observed for the sample containing only acrylamide up to $10001 / \mathrm{mm}$. At high spatial frequency $(2000 \mathrm{l} / \mathrm{mm})$ when the postprocess 2 was very fast it could be observed separately from postprocess 1 only at very short exposure times (Fig. 4b). This dependence on the spatial frequency implies that the postprocess 2 is also related to diffusion between the differently illuminated areas. Such effect 
could be expected from a diffusion of radicals or short chain polymer molecules in direction opposite to that of the monomer diffusion, i.e. from bright to dark fringe areas.

In order to check whether there could be a contribution of diffusion from the back to the front of the photopolymer layer we have measured the actual thickness of the recorded gratings by measuring their angular selectivity. It was estimated that the grating thickness is approximately $70 \%$ of the thickness of the layer and it does not depend on the recording intensity in the investigated intensity range. Even at the lowest spatial frequency of $100 \mathrm{l} / \mathrm{mm}$ the thickness of the grating is much larger than the fringe spacing. This fact combined with the pronounced dependence of the dynamics of postprocess 2 on the spatial frequency of recording supports the hypothesis that the diffusion described by postprocess 2 is in direction parallel to the grating vector.

An interesting result from data fit for the samples additionally containing crosslinker is that the decaying component can not be fitted by a single-exponential function as in the case of the samples containing one monomer only. At least a two-exponential function is necessary to fit the dynamics of postprocess 2. A detailed investigation of the influence of crosslinker on the diffusion processes after short exposure is currently in progress and will be reported elsewhere.

In order to check the hypothesis that two separate diffusion processes take place a set of gratings at constant spatial frequency were recorded. Time and intensity of recording were varied, so the final recording exposure was constant for a given set. The refractive index modulations recorded at 500 1/mm and 2000 1/mm are presented in Fig. 4a and Fig. 4b respectively. The first experimental observation is related to the maximum of the refractive index modulation. Increasing the exposure time (with an appropriate drop in intensity) produces higher diffraction efficiency of the recorded grating and hence higher refractive index modulation at both spatial 
frequencies. An explanation for such dependence can be found in the following. At longer exposure times the density of the photons in a given time interval will be smaller, so the concentration of the photoinduced radicals will be smaller. As a result the termination rate will be slower, hence a single radical will live longer and will cause the polymerization of a larger number of monomer molecules. As an ultimate result the number of the monomer molecules consumed per single photon will be higher at low intensities. Consequently at lower intensity of recording the gradient in the monomer spatial distribution will be higher and more molecules will diffuse from dark to bright fringe areas causing higher refractive index modulation. In other words, a slower polymerization rate will result in longer polymer chains and conversion of a larger number of molecules from monomer to polymer. The larger refractive index modulation at lower intensities could be therefore associated to the polymer chain length.

The second experimental observation is related to the amplitude of the initial rising component in the diffraction efficiency dynamics (see position of arrow for end of recording period). At both spatial frequencies the amplitude increases when the time of exposure is shorter and intensity is higher. This implies that when the exposure time is sufficient the main part of diffusion occurs during the recording period. Only at very short exposure times (or at very low spatial frequencies) one can observe the effect of monomer diffusion and measure the corresponding monomer diffusion time.

The third experimental observation concerns the fall off in refractive index modulation that occurs after the initial rise - postprocess 2. It is seen from Fig.4a that the amplitude of the decreasing component in the refractive index modulation is different despite the fact that the exposure is kept constant. If the time of exposure is the main factor that influences the amplitude, decreased amplitude at longer exposure times could mean that the main part of diffusion occurs 
during the recording time. Only a small part is observable after the recording is stopped. An alternative explanation could be related to the change in the intensity at shorter exposure times. At higher recording intensity the polymer chains will be shorter so they can more freely escape from bright to dark areas and in this way will decrease refractive index modulation to a greater extent. When recording at high spatial frequency - 2000 l/mm (Fig.4b) postprocess 2 becomes very fast and can be observed only at very short times of exposure (see the inner chart in Fig.4b).

Based on the data from the fitting results, the dependence of the characteristic time constants for postprocess 1 and postprocess 2 in samples containing one monomer only were extracted. As the dependence of the time constants for postprocess 1 on the spatial frequency can be discriminated only at 100 and $200 \mathrm{l} / \mathrm{mm}$, for calculation of the diffusion constant we used the data at these two spatial frequencies. The characteristic diffusion times were $0.13 \mathrm{~s}$ and $0.046 \mathrm{~s}$ for $100 \mathrm{l} / \mathrm{mm}$ and $2001 / \mathrm{mm}$ respectively. The calculated monomer diffusion constant was $\mathrm{D}_{0}=$ $1.61 \pm 0.0310^{-7} \mathrm{~cm}^{2} / \mathrm{s}$. The spatial frequency dependence of the diffusion time for postprocess 2 is shown in Fig.5. After linear fit of the data we calculated a value of $D_{0}=6.35 \pm 0.210^{-10} \mathrm{~cm}^{2} / \mathrm{s}$ for the second diffusion process. The errors were calculated after taking into account the following sources of error: error in the diffraction grating spatial frequency caused by limited accuracy of the optical set-up geometry; error in the time measurement caused by the limited time resolution; errors from the fitting procedure.

\section{Conclusions}


We have investigated the mechanism of recording in a dry acrylamide-based photopolymer system. From the measured kinetics of the refractive index modulation after small recording exposure we were able to distinguish two diffusion processes. They make opposing contributions to the final diffraction efficiency. The monomer diffusion from the dark to bright fringe areas leads to an increase of the refractive index modulation. The diffusion coefficient estimated for this process is $\mathrm{D}_{0}=1.61 \pm 0.03 .10^{-7} \mathrm{~cm}^{2} / \mathrm{s}$. It is significantly faster than other photopolymer systems known for their good response at high spatial frequency (Dupont Omnidex photopolymer for reflection holography is characterized by diffusion constant $\left.6.51 .10^{-11} \mathrm{~cm}^{2} / \mathrm{s}[11]\right)$. We ascribe the second diffusion process to diffusion of short polymer chains and/or radicals from bright to dark fringe areas with a characteristic time in order of $D_{0}=6.35 \pm 0.210^{-10} \mathrm{~cm}^{2} / \mathrm{s}$. The second diffusion process makes a negative contribution to the refractive index modulation and could be responsible for poor performance of the photopolymer at high spatial frequency of recording. Further investigations concerning the influence of the photopolymer composition (mainly the influence of the crosslinker and the molecular weight of the binder) on the diffusion rates and the photopolymer response at high spatial frequencies are in progress.

\section{Acknowledgments}

The authors thank the Facility for Optical Characterization and Spectroscopy at Dublin Institute of Technology for financial support. 


\section{References}

1. T. Trout, J. Schmieg, W. Gambogi and A. Weber, "Optical photopolymers: Design and Applictions", Adv. Mater. 10, 1219-1224 (1998).

2. S. Martin, C.A. Feely and V. Toal, "Holographic recording characteristics of an acrylamidebased photopolymer", Appl. Optics 36,5757-5768, (1997).

3. S. Guntaka, V. Toal and S. Martin, "Holographically recorded photopolymer diffractive optical element for holographic and electronic speckle-pattern interferometry”, Appl. Opt. 41, 7475-7479 (2002).

4. C. Carre and D. Lougnot, "Photopolymers for holographic recording - from standard to self-processing materials", Journal de Physique III 3 (7), 1445-1460 JUL (1993).

5. D. Psaltis and G. Burr, "Holographic data storage”, Computer Feb., 52-60 (1998).

6. S. Orlic, S. Ulm and H. Eichler, "3D bit-oriented optical storage in photopolymers" J. Opt. A: Pure and Appl. Opt. 3, 72-81 (2001).

7. L. Dhar, A. Hale, H. Katz, L.Schilling, M. Schnoes and F. Schilling, Opt. Lett. 24, 487-489 (1999).

8. S. Martin, “A new photopolymer recording material for holographic applications: Photochemical and holographic studies towards an optimized system", Ph.D. Thesis, School of Physics, (Dublin Institute of Technology, 1995).

9. J. Gallo and C. Veber, "Model of the effect of material shrinkage on volume holograms", Appl. Opt. 33, 6797-6804 (1994).

10. G. Zhao and P. Mourolis, "Diffusion model of hologram formation in dry photopolymer materials", J. Mod. Opt. 41, 1929-1939 (1994). 
11. V. Moreau, Y. Renotte and Y. Lion, "Characterisation of DuPont photopolymer: determination of kinetic parameters in a diffusion model”, Appl. Opt. 41, 3427-3435 (2002).

12. V. Colvin, R. Larson, A. Harris and M. Schilling, "Quantitative model of volume hologram formation in photopolymers", J. Appl. Phys. 81,5913-5923 (1997).

13. J. H. Kwon, H. C. Hwang and K. C. Woo, "Analysis of temporal behaviour of beams diffracted by volume gratings formed in photopolymers “, J. Opt. Soc. Am. B 16, 16511657 (1999).

14. S. Piazzolla and B. Jenkins, "First harmonic diffusion model for holographic grating formation in photopolymers”, J. Opt. Soc. Am. B 17, 1147-1157 (2000).

15. J. Lawrence, F. O’Neill and J. Sheridan, “Adjusted intensity nonlocal diffusion model of photopolymer grating formation”, J. Opt. Soc. Am. B 19, 621-629 (2002).

16. H. Kogelnik, Bell Syst. Tech. J. 48, 2909-2947, (1969).

17. B. L. Booth, "Photopolymer material for holography”, Appl. Optics, 14, 593-601 (1975).

18. W. Colburn and K. Haines, "Volume hologram formation in photopolymer materials", Appl. Opt. 10 (7), 1636-1641, (1971).

19. W. Tomlinson and E. Chandross, "Organic photochemical refractive index image recording systems”, Adv. Photochem. 12, T.N. Pitts, G. S Hammond, K. Gallnik and D. Grosjier, eds. (John Wiley Interscience 1980), pp. 201-280.

20. P. Hariharan, Optical Holography, Cambridge Studies in Modern Optics, Cambridge University Press, 1996.

21. M.G. Moharam, T.K. Gaylord, R. Magnusson, "Bragg diffraction of finite beams by thick gratings”, J. Opt. Soc. Am, 70 (3), 300- 304, 1980. 


\section{Figure captions:}

Fig.1 Refractive index modulation after recording for $0.1 \mathrm{~s}$ with intensity $40 \mathrm{~mW} / \mathrm{cm}^{2}$ at spatial frequency $200 \mathrm{l} / \mathrm{mm}$. The grating is illuminated with a homogeneous light $325 \mathrm{~s}$ after the beginning of the recording.

Fig.2 Comparison of the dynamics of refractive indexes modulation at low spatial frequencies $1001 / \mathrm{mm}$ (gray) and 2001/mm (black). Recording intensity $-40 \mathrm{~mW} / \mathrm{cm}^{2}$; recording time $-0.1 \mathrm{~s}$.

Fig.3 Spatial frequency dependence of the refractive index modulation after recording for $0.2 \mathrm{~s}$ with intensity $10 \mathrm{~mW} / \mathrm{cm}^{2}$ at $200 \mathrm{l} / \mathrm{mm}$ (black), 350 1/mm (light gray) and 500 1/mm (gray). The sample contains acrylamide and N,N Methylene bisacrylamide in 3/2 weight ratio.

Fig.4 Refractive index dynamics at constant exposure of recording: time of recording - $2 \mathrm{~s}$, recording intensity $-3.5 \mathrm{~mW} / \mathrm{cm}^{2}$ (black); $0.5 \mathrm{~s}$ and $14 \mathrm{~mW} / \mathrm{cm}^{2}$ (light gray); $0.1 \mathrm{~s}$ and $70 \mathrm{~m} \mathrm{~W} / \mathrm{cm}^{2}$ (gray). The gratings are recorded at $532 \mathrm{~nm}$ and probed at $633 \mathrm{~nm}$. Spatial frequency is $500 \mathrm{l} / \mathrm{mm}$ (a) and $2000 \mathrm{l} / \mathrm{mm}(\mathrm{b})$.

Fig.5 Dependence of characteristic time for postprocess 2 on the fringe spacing. Data for the diffusion times are extracted after fit of the recorded refractive index modulations. The gratings are recorded for $0.5 \mathrm{~s}$ with overall intensity $10 \mathrm{~mW} / \mathrm{cm}^{2}$. The recording wavelength is $532 \mathrm{~nm}$, probing wavelength $-633 \mathrm{~nm}$. Error bars are within the size of the symbols. 


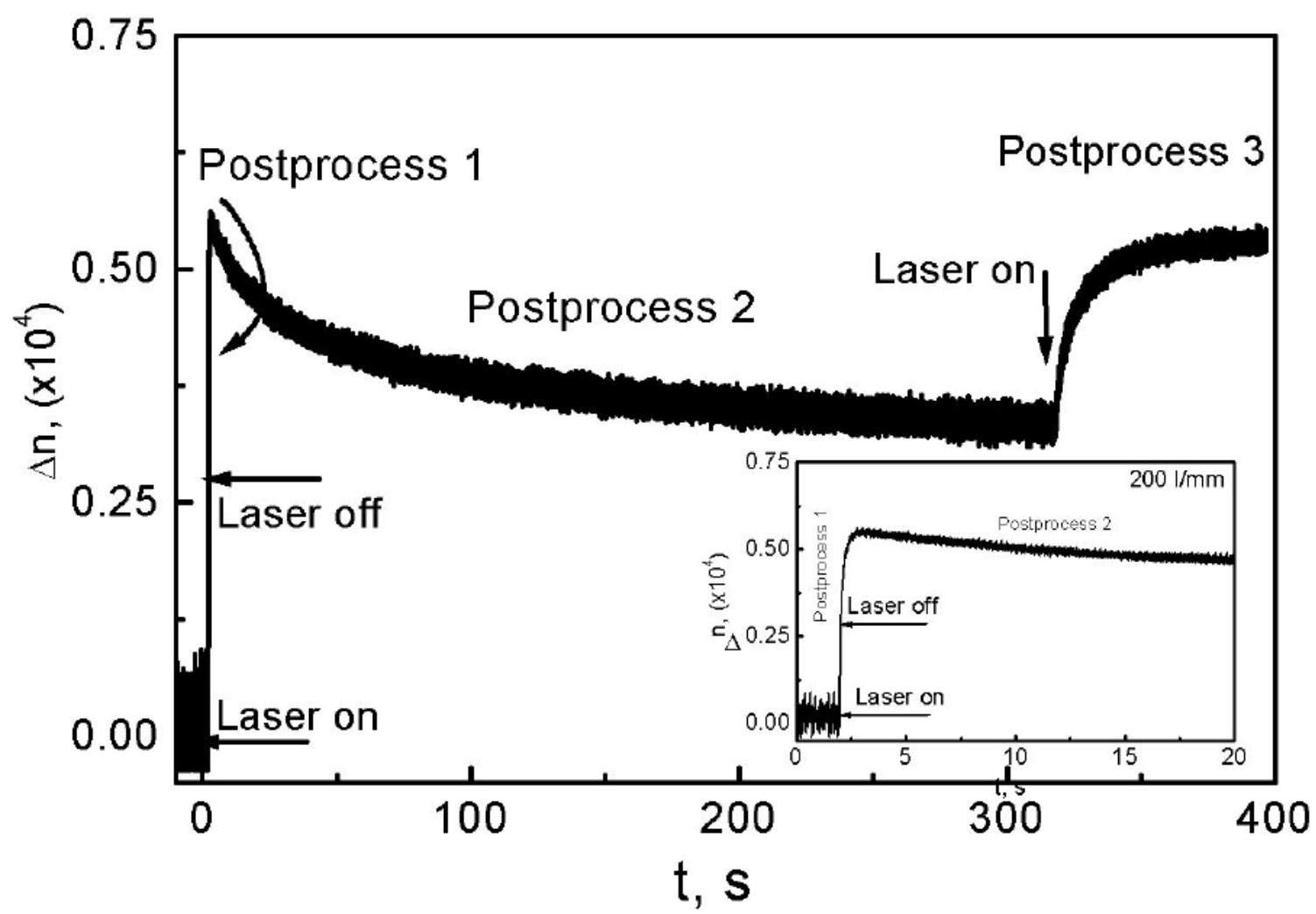

Fig.1 Refractive index modulation after recording for $0.1 \mathrm{~s}$ with intensity $40 \mathrm{~mW} / \mathrm{cm}^{2}$ at spatial frequency $200 \mathrm{l} / \mathrm{mm}$. The grating is illuminated with a homogeneous light $320 \mathrm{~s}$ after the beginning of the recording. 


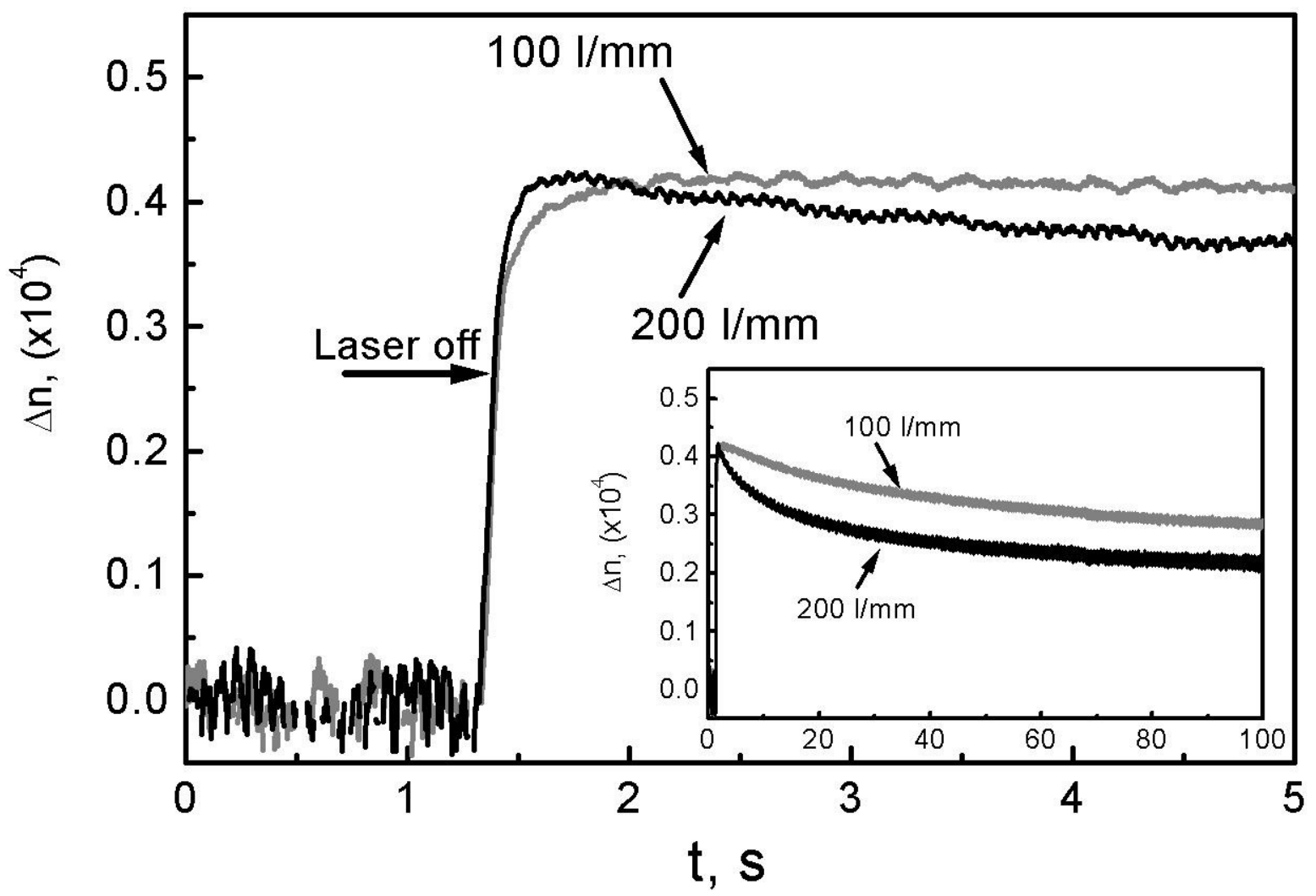

Fig.2 Comparison of the dynamics of refractive indexes modulations at low spatial frequencies - 1001/mm (gray) and 2001/mm (black). Recording intensity $-40 \mathrm{~mW} / \mathrm{cm}^{2}$; recording time $-0.1 \mathrm{~s}$. 


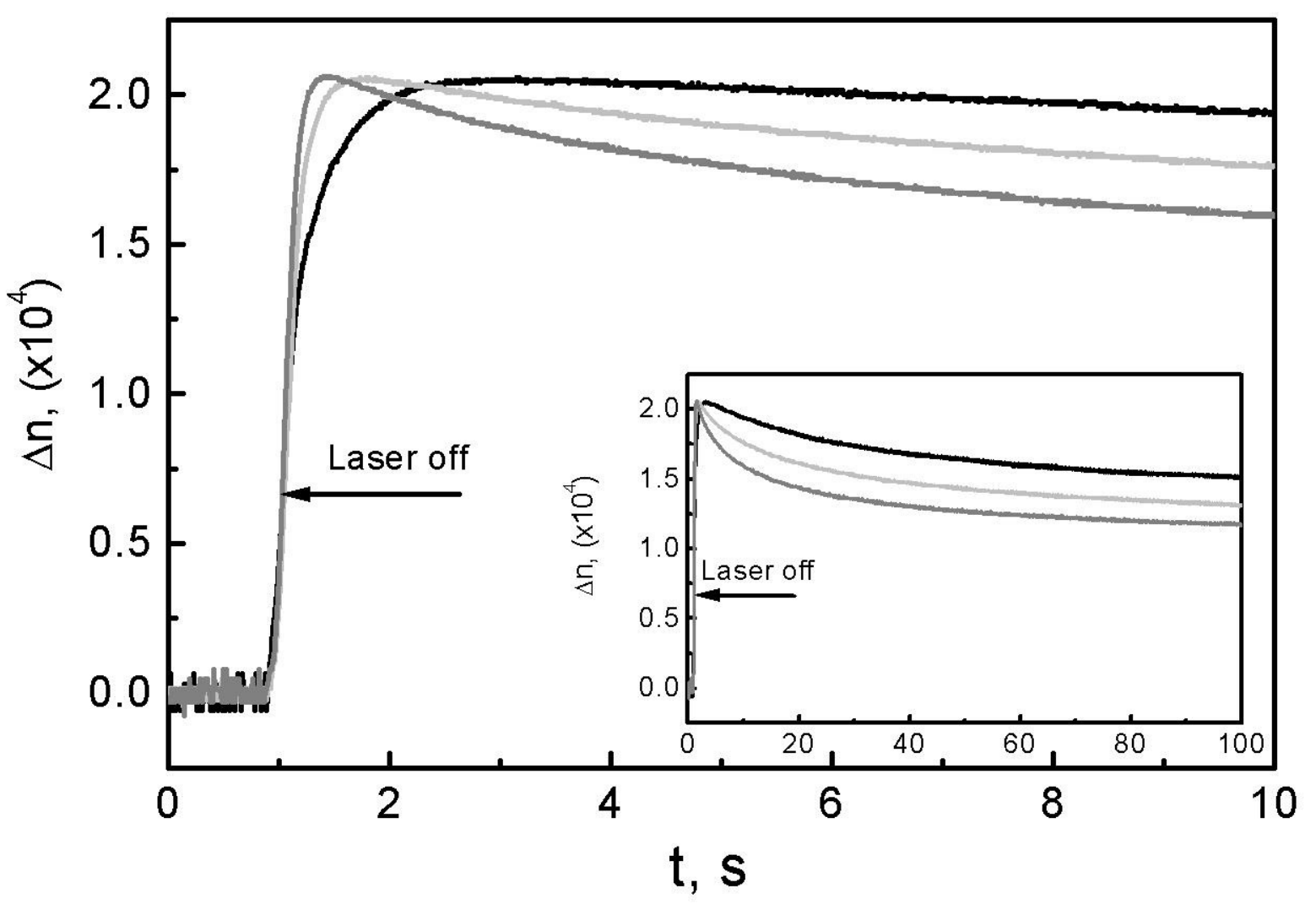

Fig.3 Spatial frequency dependence of the refractive index modulation after recording for $0.2 \mathrm{~s}$ with intensity $10 \mathrm{~mW} / \mathrm{cm}^{2}$ at $200 \mathrm{l} / \mathrm{mm}$ (black), 350 1/mm (light gray) and 500 1/mm (gray). The sample contains acrylamide and N,N Methylene bisacrylamide in $3 / 2$ weight ratio. 


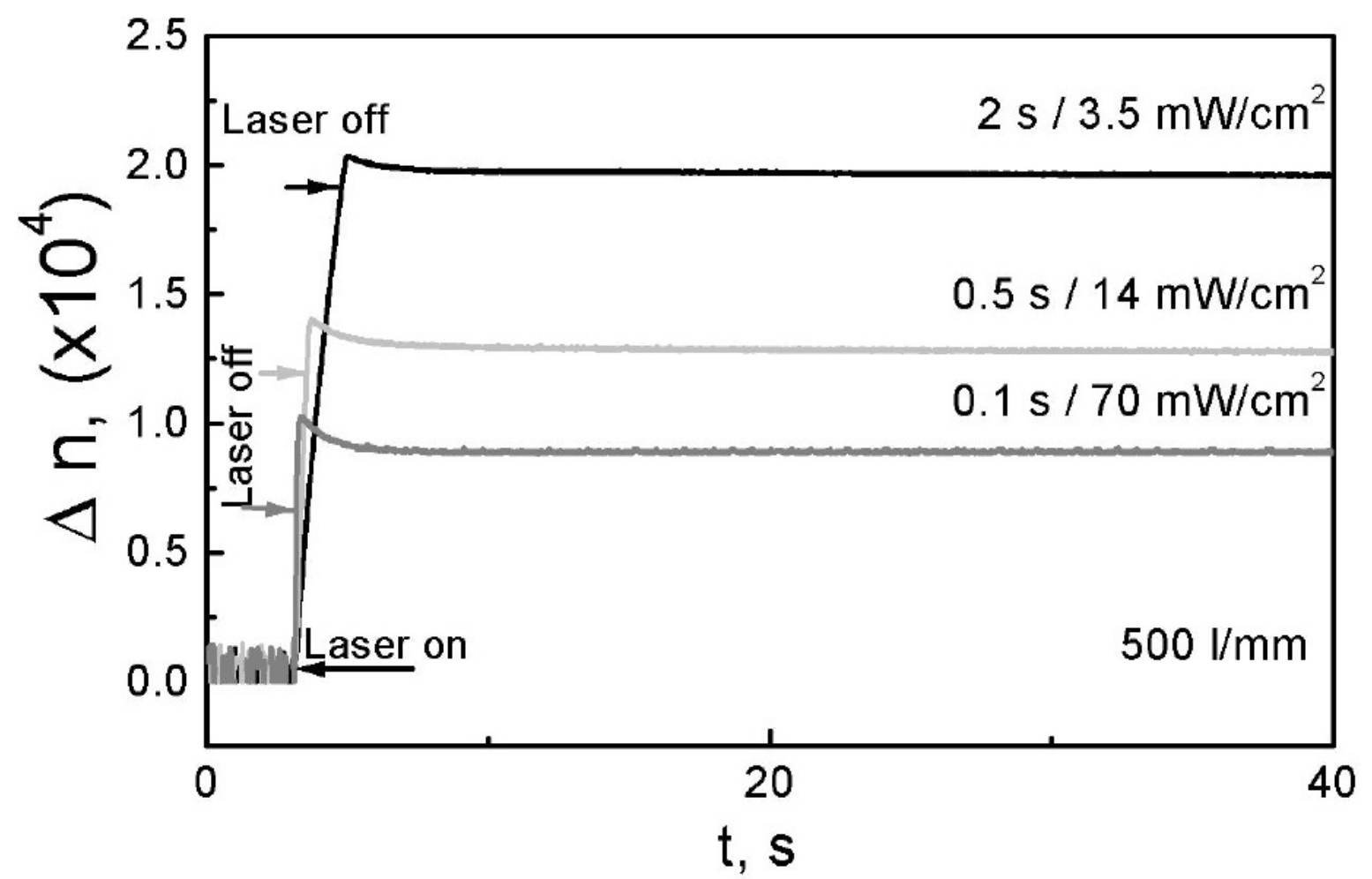

Fig.4

Fig. 4a Refractive index dynamics at constant exposure of recording: time of recording $-2 \mathrm{~s}$, recording intensity $-3.5 \mathrm{~mW} / \mathrm{cm}^{2}$ (black); $0.5 \mathrm{~s}$ and $14 \mathrm{~mW} / \mathrm{cm}^{2}$ (light gray); $0.1 \mathrm{~s}$ and $70 \mathrm{~m} \mathrm{~W} / \mathrm{cm}^{2}$ (gray). The gratings are recorded at $532 \mathrm{~nm}$ and probed at $633 \mathrm{~nm}$. Spatial frequency is $500 \mathrm{l} / \mathrm{mm}$ (a) and $2000 \mathrm{l} / \mathrm{mm}$ (b). 


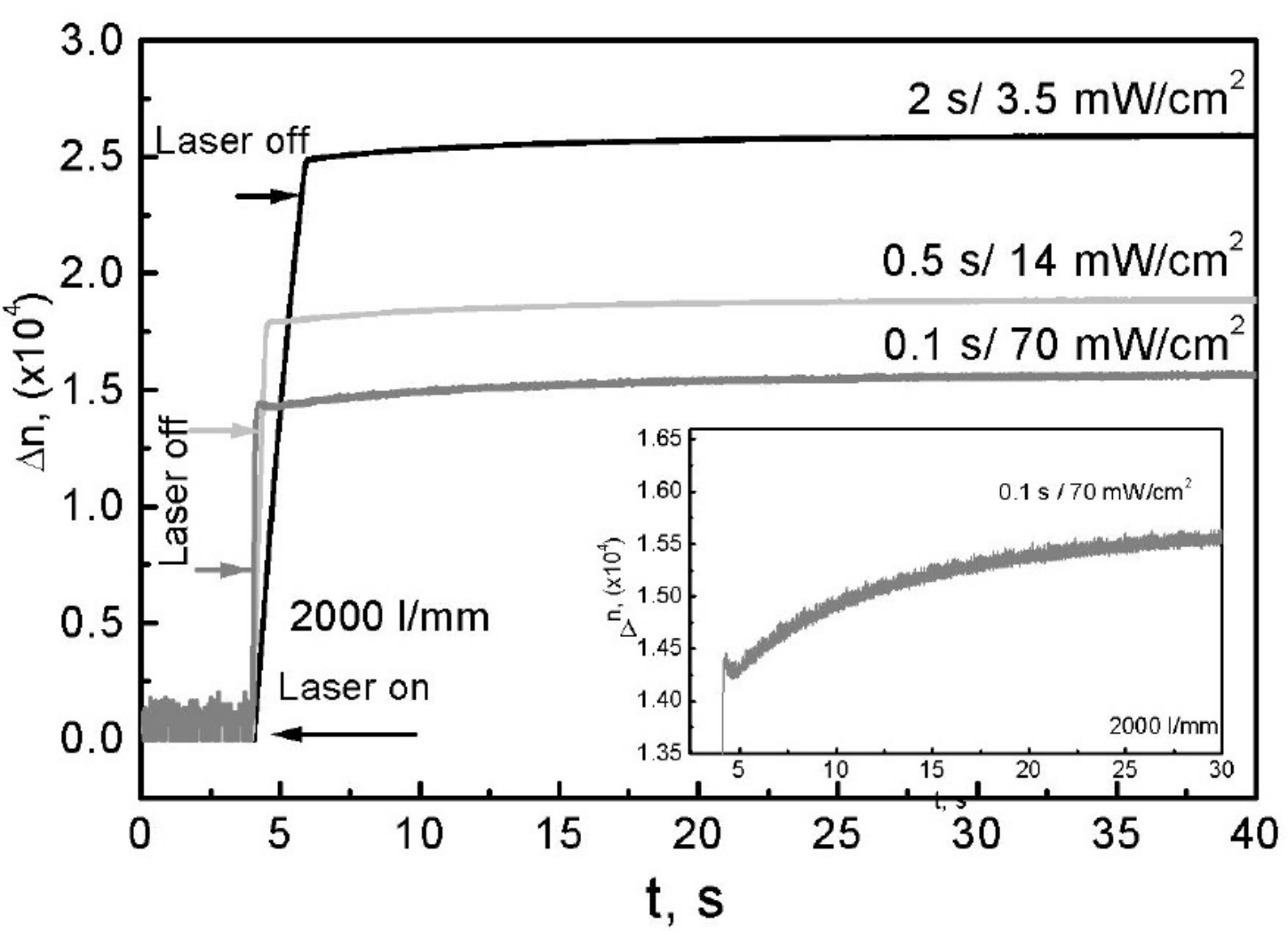

Fig.4b

Fig.4b Refractive index dynamics at constant exposure of recording: time of recording - 2s, recording intensity $-3.5 \mathrm{~mW} / \mathrm{cm}^{2}(\mathrm{black}$ ); $0.5 \mathrm{~s}$ and $14 \mathrm{~mW} / \mathrm{cm}^{2}$ (light gray); $0.1 \mathrm{~s}$ and $70 \mathrm{~m} \mathrm{~W} / \mathrm{cm}^{2}$ (gray). The gratings are recorded at $532 \mathrm{~nm}$ and probed at $633 \mathrm{~nm}$. Spatial frequency is $500 \mathrm{l} / \mathrm{mm}$ (a) and $2000 \mathrm{l} / \mathrm{mm}$ (b). 


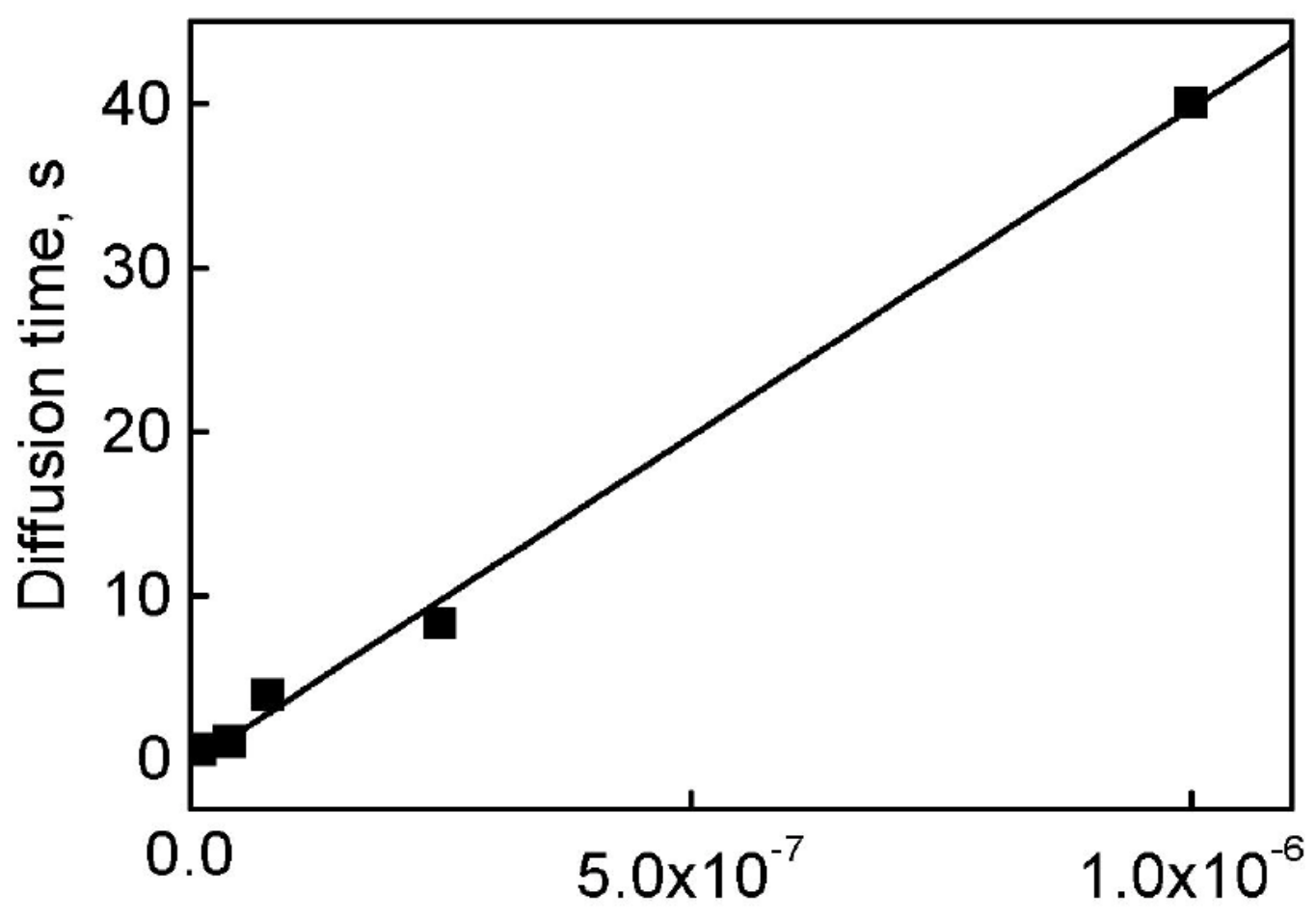

Fringe spacing, $\left(\Lambda^{2}\right) \mathrm{cm}^{2}$

Fig.5 Dependence of characteristic time for postprocess 2 on the fringe spacing. Data for the diffusion times are extracted after fit of the recorded refractive index modulations. The gratings are recorded for $0.5 \mathrm{~s}$ with overall intensity $10 \mathrm{~mW} / \mathrm{cm}^{2}$. The recording wavelength is $532 \mathrm{~nm}$, probing wavelength $-633 \mathrm{~nm}$. Error bars are within the size of the symbols. 